\title{
Effect of Fucoxanthin on PI3K/Akt Signaling Pathway in Human Cervical Cancer HeLa Cells
}

\author{
Guo Liu YE*, Li Jie JIN, Ling Ling WANG and Dan Li DU* \\ Department of Obstetrics and Gynecology, The First Affiliated Hospital of Bengbu Medical College, China
}

Received: 阱: September 27, 2018; Published:

*Corresponding author: GuoLiu YE and DanLi DU, Department of Obstetrics and Gynecology, The First Affiliated Hospital of Bengbu Medical College, Bengbu, China

Abstract

Objective: To study the effect of Fucoxanthin on PI3K / Akt signaling pathway in human cervical cancer Hela cells.

Method: We exposed HeLa cells to different concentrations of Fucoxanthin for 24 hours. The effect of Fucoxanthin on PI3K/Akt signaling pathway related proteins was detected by Western blot. In order to analyze the relationship between nuclear transcription factor activity and Fucoxanthin, the activity of NF-kappa B and AP-1 was determined by luciferase reporter gene assay.

Results: Western blot analysis showed that Fucoxanthin inactivated Akt pathway, inhibited Bcl-2 protein level, induced Bax production and caspase- 3 cleavage. The Fucoxanthin may inhibit the growth of HeLa cells by inhibiting the PI3K/Akt signaling pathway. Fucoxanthin dose dependently reduced the activation level of NF- kappa B but did not significantly affect AP-1. This indicates that Fucoxanthin inhibits the activation of NF-kappa B in HeLa cells.

Conclusion: NF-kappa B may be the mediator of Fucoxanthin-induced apoptosis in HeLa cells. As an ideal target for tumor therapy, NF-kappa B can regulate some genes involved in the development and progression of cervical cancer and inhibit cell apoptosis by activating several antiapoptosis genes.

Keywords: Fucoxanthin; cervical cancer; PI3K/Akt; Hela cells

\section{Introduction}

Epidemiological studies have found that cervical cancer is closely related to human papilloma virus (HPV) infection, probably due to genetic damage of cells and integration of high-risk HPVDNA [1]. Studies have shown that PI3K/Akt signal is over-activated in various human squamous cell carcinomas associated with HPV infection. This conclusion has been further confirmed in cervical cancer. Abnormal activation of this pathway and HPV-encoded E-series carcinogens have been reported to play a synergistic role in promoting malignant transformation of cervical cells [2]. transfected cervical cancer cells with E5 gene and found that E5 protein could up-regulate the expression of vascular endothelial growth factor by activating PI3K/Akt signal [3]. Transfected cervical cancer HeLa cells with lentiviral vector carrying siRNA of HPV18-E6 gene. It was found that inhibition of E6 protein expression could significantly down-regulate Akt expression at mRNA and protein levels, effectively inhibiting the growth of HeLa cells [4].

It was also found that over-activation of mTOR could inactivate the phosphorylation of $4 \mathrm{EBPl}$, the downstream target of mTOR, up-regulate the level of E7 mRNA cap-dependent translation and maintain the high expression of E7 protein. In turn, E7 protein could increase the level of Akt phosphorylation and maintain the survival of tumor cells by inhibiting Bax, the precursor of apoptosis
(Figure 1). PI3K/Akt/mTOR signal transduction pathway and gene changes and mutations are the fundamental causes of malignant tumors. Gene mutations related to cell growth regulation are particularly common in tumorigenesis. Some studies have shown that activation of PI3K/Akt signaling pathway can cause serious imbalance in the spectrum of many human tumors, such as ovarian cancer, breast cancer, malignant glioma, endometrial cancer, thus affecting a variety of downstream effector molecules. Activation of the PI3K/Akt pathway is also associated with the metabolic disturbance of cervical cancer [5] (Table 1).

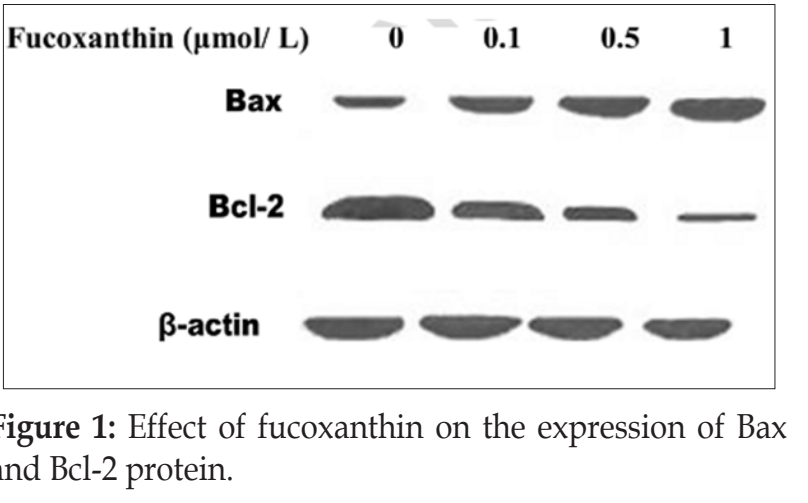


Table 1: Effect of fucoxanthin on the expression of Bax and Bcl-2 protein.

\begin{tabular}{|c|c|c|}
\hline Fucoxanthin concentration & Expression of Bcl-2 protein & Expression of Bax protein \\
\hline Contral & $0.95 \pm 0.15$ & $0.16 \pm 0.12$ \\
\hline $0.1 \mathrm{umol} / \mathrm{L}$ & $0.86 \pm 0.15^{*}$ & $0.36 \pm 0.20^{*}$ \\
\hline $0.5 \mathrm{umol} / \mathrm{L}$ & $0.46 \pm 0.23^{*} \#$ & $0.76 \pm 0.12^{*} \#$ \\
\hline $1.0 \mathrm{umol} / \mathrm{L}$ & $0.36 \pm 0.18^{* *} \$$ & $0.97 \pm 0.11^{* *} \$$ \\
\hline
\end{tabular}

Note: *Compared with the blank control group $\mathrm{P}<0.05$ ** and the blank control group $\mathrm{P}<0.01$ \# and 0.1 umol $/ \mathrm{L}, \mathrm{P}<0.05, \$$ and $0.5 \mathrm{umol} / \mathrm{L}, \mathrm{P}<0.05$ (the same below) The value in the table is the ratio of the optical density integral of the detected protein to the beta -actin (the same below).

Lee and his colleagues reported that the use of PI3K inhibitor LY294002 increased the in vitro radiosensitivity of cervical cancer cell lines [6]. NF-kappa B is a protein complex that controls gene transcription. NF-kappa B is activated in many cancers, including inflammation, transformation, proliferation, and angiogenesis. NFkappa B mediates the inhibition of apoptosis by biological signals and promotes the growth of tumor cells. The dimer of nuclear transcription factor AP-1, Fos and Jun proteins has a wide range of astonishing effects on cell proliferation and apoptosis [7] (Table 2).
PI3K/Akt/mTOR signaling pathway plays an important role in the development and treatment of cervical cancer. As a new target of tumor therapy, the related research is not enough. Therefore, it is worthwhile to further clarify the role of PI3K/Akt pathway and its gene expression in the pathogenesis of cervical cancer, to explore the regulation of PI3K/Akt pathway and its interaction with other pathways, to determine the most effective therapeutic targets and methods, and to search for ideal antitumor drugs.

Table 2: Effect of fucoxanthin on the expression of Caspase-3protein.

\begin{tabular}{|c|c|}
\hline Fucoxanthin concentration & Expression of caspase-3 protein \\
\hline Contral & $0.10 \pm 0.12$ \\
\hline $0.1 \mathrm{umol} / \mathrm{L}$ & $0.26 \pm 0.20$ \\
\hline $0.5 \mathrm{umol} / \mathrm{L}$ & $0.67 \pm 0.12^{*} \#$ \\
\hline $1.0 \mathrm{umol} / \mathrm{L}$ & $0.89 \pm 0.11^{* *} \$$ \\
\hline
\end{tabular}

Note: * Compared with the blank control group $\mathrm{P}<0.05$ ** and the blank control group $\mathrm{P}<0.01$ \# and 0.1 umol $/ \mathrm{L}, \mathrm{P}<0.05$, \$and $0.5 \mathrm{umol} / \mathrm{L}, \mathrm{P}<0.05$ (the same below) The value in the table is the ratio of the optical density integral of the detected protein to the beta -actin (the same below).

\section{Materials and Methods}

\section{Chemicals and Medicines:}

Fucoxanthin (purity (>99.68\%) was purchased from Anhui University of Traditional Chinese Medicine (Hefei, China). It was suspended in 5\% sodium carboxymethyl cellulose solution before use. Other chemicals are at the highest level. Cell culture reagents come from Gibco life science and Technology Corporation (NY, USA).

\section{Cell Culture:}

\section{cleaved caspase-3}

\section{B-actin}

Figure 2: Effect of fucoxanthin on the expression of Caspase-3protein.

HeLa cells are obtained from human cervical cancer cells and are derived from the American Model Culture Collection (ATCC,
Manassas, Virginia, USA). The cells grew in RPMI 1640 medium containing 10\% fetal bovine serum (FBS), $10 \mathrm{mmol} / \mathrm{L}$ hydroxyethyl piperazine ethylsulfonic acid, $2 \mathrm{mmol} / \mathrm{L}$-glutamine, $50 \mathrm{mmol} / \mathrm{L}$ beta-mercaptoethanol, $1 \mathrm{mmol} / \mathrm{L}$ sodium pyruvate, $100 \mathrm{U} / \mathrm{ml}$ penicillin and $100 \mathrm{mmol} / \mathrm{ml}$ streptomycin, and 95\% empty at 37 0C (Figure 2). A humid environment of gas and 5\% carbon dioxide; exposes cells to various concentrations of Fucoxanthin for a specified period of time.

\section{Western Blot Method:}

First extract the total protein of HeLa cells from different groups after treated with Fucoxanthin by PMSF lysate; after electrophoresis, the proteins in the tested samples were divided into different bands according to the large molecular weight of the protein in the gel samples; after that, the desired target bands were transferred to the PVDF membrane; the indirect immunoenzyme standard was used. The method is chem luminescence and darkroom development (Table 3).

Table 3: Effect of fucoxanthin on the expression of PI3K/Akt protein.

\begin{tabular}{|c|c|c|}
\hline Fucoxanthin concentration & ExpressionPI3K protein & Expression of Akt protein \\
\hline Contral & $0.65 \pm 0.15$ & $0.96 \pm 0.12$ \\
\hline $0.1 \mathrm{umol} / \mathrm{L}$ & $0.56 \pm 0.15^{*}$ & $0.84 \pm 0.20$ \\
\hline $0.5 \mathrm{umol} / \mathrm{L}$ & $0.32 \pm 0.23^{*} \#$ & $0.66 \pm 0.12^{*} \#$ \\
\hline $1.0 \mathrm{umol} / \mathrm{L}$ & $0.15 \pm 0.18^{*} \$$ & $0.37 \pm 0.11^{* *} \$$ \\
\hline
\end{tabular}


Transient Transfection and Luciferase Reporter Gene Assay:

Cells were transfected with pGL3.5X NF-kappa B-1uciferase reportergene plasmid and RL-TK-Renillaluciferase reporter plasmid to stimulate the corresponding time point, and the transcriptional activity of NF-kappa B reporter gene was determined by DLR (Dualluciferase reporter assay system, Promega) (Figure 3). Reference kit instructions for the detection methods were summarized as follows: cells treated with different methods were washed with cold PBS twice, residual PBS was absorbed, PLB lysate (24-well plate) was added, cells were lysed at room temperature for 15 minutes, and the samples were preserved at-80C. The cold light value of NF-kappa B-firefly (Berthold Technology, Lumat 9506 Cold Light Detection System) was measured immediately after adding 20UL LAR to the 4UL sample, and then adding 20 UL Stop-Glo reagent to activate Renilla fluorescence while inactivating firefly fluorescence. After shaking and mixing, the cold light value of pRL-TK Renilla was measured immediately. The ratio of the cold light value of NF-kappa B firefly to the cold light value of pRL-TK-Renilla was recorded as the NF-kappa reporter gene activity value (pRL-TK-Renilla cold light value was used to balance the transfection efficiency differences between the different pores).

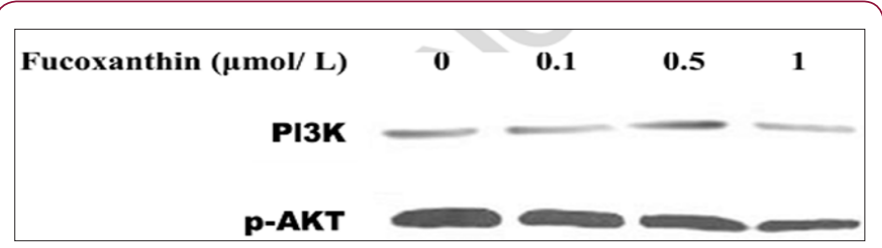

Figure 3: Effect of fucoxanthin on the expression of PI3K Akt protein.

\section{Statistical Processing}

Data were input into computer and processed with Microsoft Excel 2003 software and SPSS 22 software. T-test showed that the difference was significant when $P$ value was less than 0.05 and $P$ value was less than 0.01 .

\section{Results}

\section{Determination of Western Blot}

Effect of on the Expression of Bcl-2 and Bax Protein: The results showed that the expression of Bcl-2 decreased and that of Bax increased with the prolongation of action time. The apoptosis of HeLa cells could be induced by fucoxanthin of Bax to Bcl-2, which was time-dependent.

The Influence on Caspase-3: This study showed that fucoxanthin could increase the activity of Caspase-3 in HeLa cells, and the activity increased with the prolongation of drug action time. It is suggested that fucoxanthin may also induce apoptosis through caspase-3 pathway.

Influence on P13K / Akt: The results showed that after 24 hours, P13K decreased by $13.0 \%, 50.1 \%$ and $76.9 \%$ respectively, and Akt decreased by $12.5 \%, 31.2 \%$ and $61.5 \%$ respectively, which indicated that fucoxanthin could inhibit PI3K/Akt signal transduction pathway. This study confirmed that fucoxanthin could also induce apoptosis of HeLa cells. In this way, the PI3K/Akt signal transduction pathway may directly induce apoptosis of HeLa cells. The role of fucoxanthin is extensive, and its specific mechanism needs further study. luciferase reporter assay results

In order to analyze the relationship between nuclear transcription factor activity and fucoxanthin, the activity of NFkappa B and AP-1 was determined by luciferase reporter gene method. The results showed that fucoxanthin dose-dependent decreased the activation level of NF-kappa B (Figure 4) but had no significant effect on AP-1 (Figure 5).

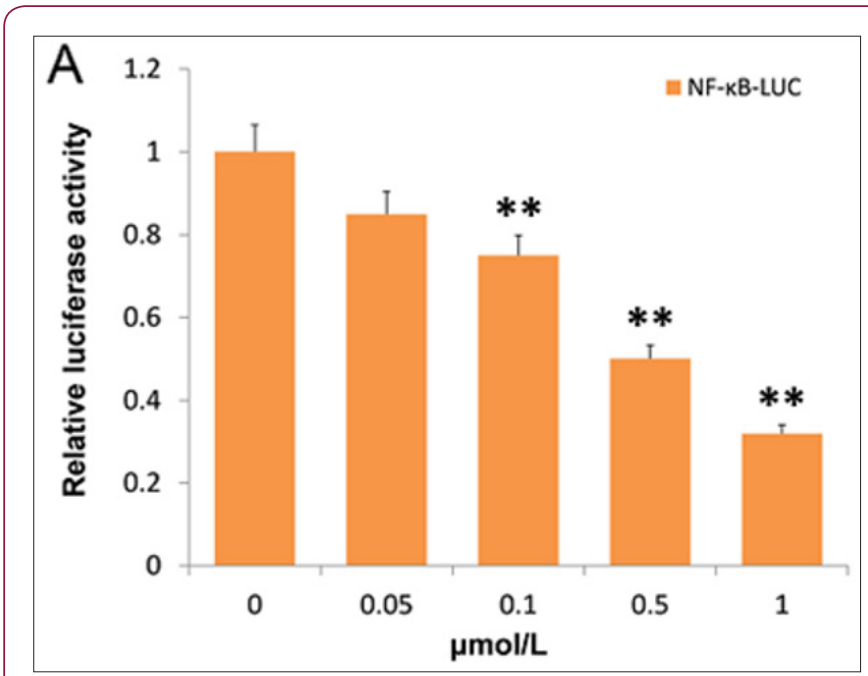

Figure 4: fucoxanthin dose dependently decreased activation of NF-kB.

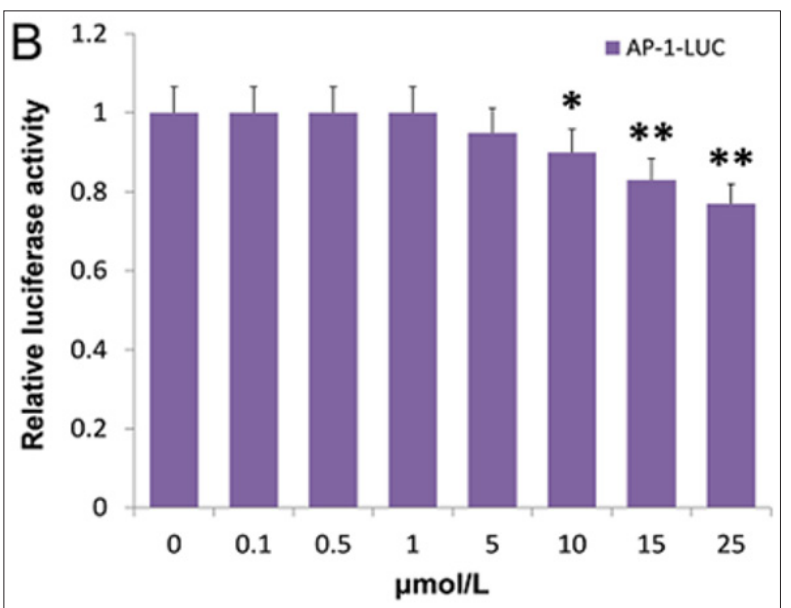

Figure 5: Effect of fucoxanthin on AP-1 activity.

\section{Detect NF- kappa B by Western blot.}

fucoxanthin inhibits the transfer of NF-kappa B from cytoplasm to nucleus and increases the protein level of NF-kappa B (P65) in cytoplasm. These data suggest that NF- kappa B may be the vector for HeLa cells apoptosis induced by algal yellow substance.

\section{Discussion}

Above studies showed that the cells were exposed to different concentrations of Fucoxanthin for 24 hours. Western blot analysis 
showed that Fucoxanthin inactivated the Akt pathway, inhibited the protein level of Bcl-2, induced Bax production and caspase- 3 cleavage (Figure 6). Therefore, the results suggest that Fucoxanthin may inhibit the growth of HeLa cells by inhibiting PI3K/Akt signaling pathway. Fucoxanthin inhibits the activation of NF-kappa B in HeLa cells. As an ideal target for tumor therapy, NF-kappa B can regulate some genes involved in the development and progression of cancer and inhibit cell apoptosis by activating several anti-apoptotic genes [8-10]. The induction and execution of apoptosis requires the coordination of a series of molecules, such as signal molecules, receptors, enzymes and gene-regulated proteins. Caspase protease family is considered to play a key role in the process of apoptosis. Caspase (cysteine aspartate-specific protease) belongs to cysteine protease, which is the key enzyme causing cell apoptosis.

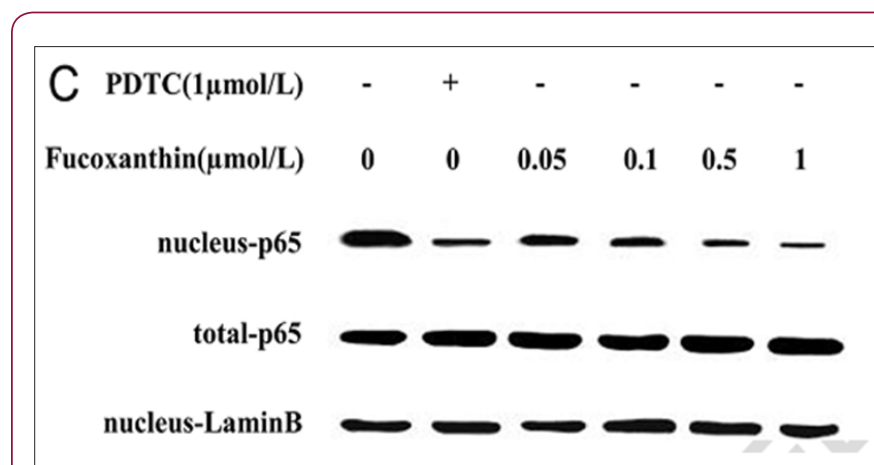

Figure 6: Western blot detection of fucoxanthin effect cytoplasm and nuclei protein leve of NF- $\mathrm{kB}$.

Caspase exists as an inactive proenzyme in cells. Under the regulation of apoptotic signals, the precursor pro-caspase is cleaved to produce two active subunits, which then triggers a series of caspase cascade amplification signal transduction processes [11]. Up to now, 14 caspases have been found, of which at least 8 caspases are associated with apoptosis [12]. They are usually classified into two broad categories: initial caspases, including Caspase-2, $-8,-9$, and-10; and execution caspases, including Caspase-3, -6, -7. Caspase- 8 and -9 activate the death receptor pathway and mitochondrial pathway respectively [13]. Inducing apoptosis of tumor cells is an important strategy for tumor therapy. Most tumor therapies activate the apoptotic process of tumor cells to kill tumor cells. Therefore, it is an effective way to find out the exact molecular mechanism of tumor cell apoptosis and the apoptosis pathway of tumor cells treated by drugs. Abnormal activation of PI3K/Ak $t$ pathway is thought to be important for the mechanisms of tumor cell escape from apoptosis, abnormal proliferation and tumor angiogenesis [14].

It is suggested that PI 3K/Akt signaling pathway may regulate apoptosis by regulating the activity of bc1-2 family members, inhibiting caspase's pro-apoptotic function and activating transcription factor NF-kappa B to promote cell proliferation [1516]. Specifically, activation of PI3K/Akt pathway can depolymerize $\mathrm{Bcl}-2$ and make free Bcl-2 play its anti-apoptotic role; directly catalyze Caspase-3 and Caspase-9's Serl96 to inactivate it, and cause cells to proliferate excessively by inhibiting the apoptotic function of caspase; activate transcription factor NF-kappa B to promote it.
After entering the nucleus, it inhibits cell apoptosis and promotes cell proliferation [17]. PI3K is a kind of phosphatidy linositol kinase, which catalyzes phosphorylation of groups, including PI3K, P14K and P15K. It is a key signal molecule in many life activities. It can mediate signal transduction pathways to regulate cell division, differentiation and apoptosis. The molecular weight of PI3K is 85KD. It is for the catalytic subunit P110 of $110 \mathrm{KD}$ [18].

Akt gene is a serine/threonine protein kinase, often called protein kinase B. It is a direct target protein downstream of P13K and can be directly activated by PI3K. HKT family members include Akt-1, Akt-2 and Akt-3 [19]. They are widely expressed in various tissues and have similar protein structures, but they are regulated by completely different gene coding. The amplification and over expression of Akt gene can be detected in a large number of human tumor cells. Studies have shown that the expression of phosphorylated Akt protein in cervical cancer is associated with the occurrence and development of cervical cancer and can be used as an independent risk factor for predicting adverse prognosis of patients [20]. The anti-apoptosis effect of PI3K/Akt signaling pathway may be related to the following mechanisms:

a) Regulating the activity of Bcl-2 family members. Bcl-2 family members are divided into two types: apoptosis inhibitory protein and pro apoptotic protein. Bcl-2, bcl-xL, bcl-G, MCL-1 and Bax, Bad and Bak can inhibit cell apoptosis. The formation and balance of homologous dimer and heterodimer of Bcl-2 family is the key to cell survival or apoptosis. Activation of PI3Kdependent Akt can phosphorylate Ser136/Ser112 residues of Bad, depolymerize phosphorylated Bad with Bcl-2 or bcl-xL, and bind Bad with anti-apoptotic binding protein, while free Bcl-2 plays an anti-apoptotic role [21]. At the same time, activation of PI3K/Akt pathway can inactivate the phosphorylation of Bax's Ser184 residue and inhibit cell apoptosis [22].

b) Inhibit the activation of aspartic specific cysteine protease family members. Activated Akt can directly catalyze the phosphorylation of caspase- 9 to Ser196 and caspase-3, inactivate them and inhibit caspase-induced apoptosis.

c) Inhibit the activity of GSK3. Activation of GSK3 in mature cells can induce degradation of beta-catenin protein, inhibit cell adhesion and accelerate cell apoptosis. Akt may inhibit cell apoptosis after GSK3 inactivation, and at the same time, Akt activation can accelerate glycolysis, increase ATP production and inhibit cell apoptosis. Inhibition of apoptosis gene expression. Activated Akt can phosphorylate FKHR protein, promote the binding of FKHR protein and anti-apoptotic binding protein to remain in the cytoplasm, inhibit the expression of FasL induced by FKHR entering the nucleus, and thus inhibit cell apoptosis [23]. Activating transcription factor NF- kappa B. NF- kappa $B$ promotes cell proliferation and inhibits cell apoptosis after entering the nucleus. Meanwhile, NF- kappa B activates ATM/ ATR and DNA-PK to promote repair of DNA damage in cells [24]. Prevent mitochondria from releasing cytochrome $\mathrm{c}$ and apoptosis factors. When cells are damaged, because of the mitochondrial membrane. 


\section{Conclusion}

We exposed HeLa cells to different concentrations of Fucoxanthin for 24 hours. Western blot analysis showed that Fucoxanthin inactivated the Akt pathway, inhibited the protein level of Bcl-2, and induced the formation of Bax and the cleavage of caspase-3. Therefore, the results suggest that Fucoxanthin may inhibit the growth of HeLa cells by inhibiting PI3K/Akt signaling pathway. In order to analyze the relationship between nuclear transcription factor activity and Fucoxanthin, the activities of NFkappa B and AP-1 were determined by luciferase reporter gene method. The results showed that Fucoxanthin dose-dependent decreased the activation level of NF-kappa B but had no significant effect on AP-1. This indicated that Fucoxanthin inhibited the activation of NF-kappa B in HeLa cells. Western blot analysis showed that Fucoxanthin inhibited the transfer of NF-kappa B from nucleus to cytoplasm and increased the level of nuclear NF-kappa B (P65) protein. These data indicate that NF- kappa B may be a vector for HeLa cells apoptosis induced by Fucoxanthin. As an ideal target for tumor therapy, NF-kappa B can regulate some genes involved in the development and progression of cervical cancer and inhibit cell apoptosis by activating several anti-apoptosis genes.

\section{Acknowledgment}

This research was supported by Science and technology development fund of Bengbu Medical College (Bykf13A25); Natural Science Research Project Funding (KJ2016A482), Education Department of Anhui Province, China.

\section{References}

1. Kim KN, Ahn G, Heo SJ, Kang SM, Kang MC, et al. (2013) Inhibition of tumor growth in vitro and in vivo by fucoxanthin against melanoma B16F10 cells. Environ Toxicol Pharmacol 35(1): 39-46.

2. Maeda H, Hosokawa M, Sashima T, Takahashi N, Kawada T, et al. (2016) Fucoxanthin and its metabolite, fucoxanthinol, suppress adipocyte differentiation in 3T3-L1 cells. Int J Mol Med 18(1): 147-152.

3. Das SK, Hashimoto T, Shimizu K, Yoshida T, Sakai T, et al. (2015) Fucoxanthin induces cell cycle arrest at G0/G1 phase in human colon carcinoma cells through up-regulation of p21WAF1/Cip1. Biochim Biophys Acta 1726(3): 328-335.

4. Ji Y, Lu G, Chen G, Huang B, Zhang X, et al. (2017) Microcystin LR induces apoptosis via NF-kappaB/iNOS pathway in INS-1 cells. Int J Mol Sci 12(7): 4722-4734.

5. Wang Y, Zhu Y, Gao L, Yin H, Xie Z, et al. (2017) Formononetin attenuates IL-1beta-induced apoptosis and NF-kappaB activation in INS-1 cells. Molecules 17(9): 10052-10064.

6. Zhao H, Xu Z, Qin H, Gao Z, Gao L (2014) miR-30b regulates migration and invasion of human colorectal cancer via SIX1. Biochem J 460(1): 117-125.

7. Han X, Sun Y, Scott S, Bleich D (2011) Tissue inhibitor of metalloproteinase-1 prevents cytokine-mediated dysfunction and cytotoxicity in pancreatic islets and beta-cells. Diabetes 50(5): 10471055 .
8. Meng ZX, Nie J, Ling JJ, Sun JX, Zhu YX, et al. (2015) Activation of liver $\mathrm{X}$ receptors inhibits pancreatic islet beta cell proliferation through cell cycle arrest. Diabetologia 52(1): 125-135.

9. Lu G, Zheng M, Zhu Y, Sha M, Wu Y, et al. (2012) Selection of peptide inhibitor to matrix metalloproteinase- 2 using phage display and its effects on pancreatic cancer cell lines PANC-1 and CFPAC-1. Int J 4 Biol Sci 8(5): 650-662.

10. Morgensztern D, McLeod HL (2015) PI3K/Akt/mTOR pathway as a target for cancer therapy. Anticancer Drugs 16(8): 797-803.

11. Chang F, Lee JT, Navolanic PM, Steelman LS, Shelton JG, et al. (2013) Involvement of PI3K/Akt pathway in cell cycle progression, apoptosis, and neoplastic transformation: a target for cancer chemotherapy. Leukemia 17(3): 590-603.

12. Olivier S, Robe P, Bours V (2016) Can nf-kappab be a target for novel and efficient anti-cancer agents? Biochem Pharmacol 72(9): 1054-1068.

13. Greten FR, Karin M (2014) The IKK/NF-kappaB activation pathway a target for prevention and treatment of cancer. Cancer Lett 206(2): 193199.

14. Passarella RJ, Zhou L, Phillips JG, Wu H, Hallahan DE, et al. (2017) Recombinantpeptides as biomarkers for tumorresponse to molecular targeted therapy. Clin Cancer Res 15(20): 6421-6429.

15. Xue G, Restuccia DF, Lan Q Hynx D, Dirnhofer S, et al. (2012) Akt/PKBmediated phosphorylation of twist1 promotes tumor metastasis via mediating cross-talk between PI3K/Akt and TGF-beta signaling axes. Cancer Discov 2(3): 248-259.

16. Tan J, Yu Q (2013) Molecular mechanisms of tumor resistance to PI3KmTOR targeted cancer therapy. Chin J Cancer 32(7): 376-379.

17. Wander SA, Zhao D, Besser AH, Hong F, Wei J, et al. (2013) PI3K/mTOR inhibition can impair tumor invasion and metastasis in vivo despite a lack of antiproliferative action in vitro: implications for targeted therapy. Breast Cancer Res Treat 138(2): 369-381.

18. Guan P, Howell Jones R, Li N, Bruni L, de Sanjose S, et al. (2012) Human papillomavirus types in 115,789 HPV-positive women: a meta-analysis from cervical infection to cancer. Int J Cancer 131(10): 2349-2359.

19. Joo YH, Jung CK, Sun DI, Park JO, Cho KJ, et al. (2012) High-risk human papillomavirusand cervical lymphnodemetastasisinpatients with oropharyngeal cancer. Head Neck 34(1): 10-14.

20. Hisamatsu T, Mabuchi S, Yoshino K, Fujita M, Enomoto T, et al. (2012) Prediction of progression-free survival andresponse to paclitaxel plus carboplatin in patients with recurrentor advanced cervical cancer. Int J Gynecol Cancer 22(4): 476623-476629.

21. Yan X, Chuda Y, Suzuki M, Nagata T (1999) Fucoxanthin as the major antioxidant in Hijikia fusiformis, a common edible seaweed. Biosci Biotechnol Biochem 63(3): 605-607.

22. Satomi Y (2012) Fucoxanthin induces gadd45a expression and g1 arrest with SAPK/JNK activation in lncap human prostate cancer cells. Anticancer Res 32(3): 807-813.

23. Hosokawa M, Kudo M, Maeda H, Kohno H, Tanaka T, et al. (2014) Fucoxanthin induces apoptosis and enhances the antiproliferative effect of the PPARgamma ligand, troglitazone, on colon cancer cells. Biochim Biophys Acta 1675(1-3): 113-119.

24. Nah SS, Ha E, Lee HJ, Chung JH (2017) Inhibitory effects of melittin on the production of lipopolysaccharide-induced matrix metalloproteinase 3 in human osteoarthritic chondrocytes. Toxicon 49(6): 881-885. 


\section{ISSN: 2574-1241}

DOI: 10.26717/BJSTR.2018.09.001819

GuoLiu YE, DanLi DU. Biomed J Sci \& Tech Res

cC (i) This work is licensed under Creative

Submission Link: https://biomedres.us/submit-manuscript.php

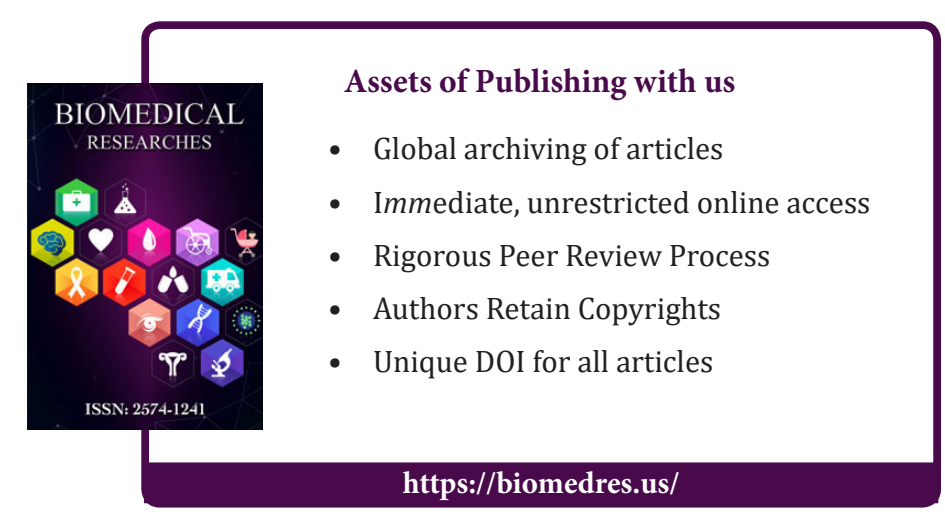

